\title{
On traces of Fourier integral operators on submanifolds *
}

\author{
P. Sipailo ${ }^{\dagger}$
}

October 9, 2018

\begin{abstract}
Given a smooth embedding $i: X \hookrightarrow M$ of manifolds and a Fourier integral operator $\Phi=\Phi(\Lambda)$ on $M$ associated with a Lagrangian submanifold $\Lambda \subset T^{*}(X \times X) \backslash\{0\}$, we consider its trace $i^{!}(\Lambda)$ on the submanifold $X$, i.e. the composition $i^{*} \Phi i_{*}$, where $i^{*}$ and $i_{*}$ are the boundary and coboundary operators, respectively. We establish the conditions under which the trace $i^{!}(\Phi)$ is also a Fourier integral operator, and calculate its amplitude in canonical local coordinates.
\end{abstract}

\section{Contents}

1 Introduction 1

2 Preliminaries 3

2.1 Fourier integral operators . . . . . . . . . . . . . 3

2.2 Traces of operators and traces of Lagrangian manifolds . . . . . . . 5

3 Traces of Fourier integral operators 6

3.1 The main theorem . . . . . . . . . . . . . . 6

3.2 Calculation of amplitude . . . . . . . . . . . . . 10

3.3 Application to quantized canonical transformations . . . . . . . . . 13

\section{Introduction}

Given a smooth embedding $i: X \hookrightarrow M$ of manifolds and an operator $A$ on $M$, the trace of $A$ (see [1,2]) is an operator denoted by $i^{!}(A)$ on the submanifold $X$ given by the composition

$$
i^{!}(A)=i^{*} A i_{*},
$$

where $i^{*}$ is the boundary operator, i.e. the operator of restriction to $X$, and $i_{*}$ is a dual coboundary operator, which is defined in a dual manner (more precisely, it takes a function on $X$ to a distribution on $M$ localized at $X$ ). The concept of trace of operator on a manifold plays a central role in the so called relative elliptic theory (see [4, 5]), i.e. a theory associated with a pair of manifolds $(M, X)$. In

*The work is supported by RFBR grant NN 16-01-00373 A.

${ }^{\dagger}$ RUDN University, Russia 
particular the trace operation is the main tool in studying the Sobolev problem, which us a pseudodifferential problem with boundary conditions posed on an embedded submanifold (see [6,7]). It was quickly discovered that the trace operation behaves fairly well within the class of pseudodifferential operators (PDOs), namely the trace of a PDO is again a PDO. But the things become more complicated if one deals with a wider class of operators. For instance, given a $G$-operator (i.e. an operator associated with an action of a group $G$ ), its trace turns out to be an operator of some very different nature: in particular (under suitable conditions), it is localized at the set of fixed points of group action, that is, it is compact outside any neighbourhood of this set (see [8]). A similar situation occurs in studying manifolds with singularities (see [9]).

A closer analysis shows that in some situations such localized operators can be described as Fourier integral operators (FIOs). Besides that FIOs naturally appear in relative elliptic theory as compositions with bundary/coboundary operators. Note also that the shift operators (building bricks for $G$-operators) are special cases of quantized canonical transformations (FIOs associated with graphs of canonical transformations), and the latter operators arise in differential equations quite widely (see also [3], where $G$-operators associated with groups of quantized canonical transformations were studied).

The above observations lead to the question whether the trace of a FIO on a submanifold (or the trace of a quantized canonical transformation) is again a FIO on this submanifold. It is easy to see that in general the answer is "no": the reason is that given a trace of a FIO, the underlying Lagrangian submanifold in the cotangent bundle may have singularities or boundary (while classical FIOs are associated with smooth (immersed) Lagrangian submanifolds). So the next natural question is to determine the conditions guaranteeing that the trace of a FIO is a FIO again. This question is the main problem of the current paper.

Let us make some remarks on the present work. First, observe that the boundary and coboundary operators are aslo special cases of FIOs, so the subject of our study is in fact just a composition of three FIOs. However, the classical theory of FIOs does not capture such type of composition, since the Lagrangian submanifolds corresponding to $i^{*}$ and $i_{*}$ do intersect the zero section in the factors of $T^{*} M \times T^{*} X \simeq T^{*} X \times T^{*} M$. Second, we note this paper is actually an extension of [10]. The differences are that now our traces are associated with general Lagrangian submanifolds (while in [10] these submanifolds were just isolated fibers of the cotangent bundle), and now we deal with clean intersections rather than with transversal ones.

Finally, let us discuss the contents of the paper. First (after recalling some basic facts about FIOs and traces of operators), we introduce the notion of trace of Lagrangian submanifold, associated with the embedding $i: X \hookrightarrow M$. This is an operation, which takes a Lagrangian submanifold $\Lambda \subset T^{*}(M \times M)$ to some submanifold of $i^{!}(\Lambda) \subset T^{*}(X \times X)$. Then we formulate the main theorem of the paper, which states that the trace of a FIO $\Phi=\Phi(\Lambda)$ associated with a Lagrangian submanifold $\Lambda$ under certain conditions is a FIO associated with $i^{!}(\Lambda)$ (and, in particular, $i^{!}(\Lambda)$ is Lagrangian). In other words, we describe the naturality of the trace operation within the class of FIOs in the sense of commutativity of the following 
diagram:

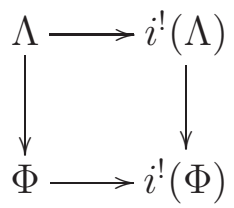

where the vertical arrows stand for the correspondence between Lagrangian manifolds and associated FIOs. After that we calculate the amplitude of the resulting FIO by means of canonical coordinates on $i^{!}(\Lambda)$. In the last section we discuss a special case of quantized canonical transformations.

The author is grateful to Prof. B.Yu.Sternin and to Prof. A.Yu.Savin for the support and deep attention to his work.

\section{Preliminaries}

Throughout the paper, the subscript " 0 " means removing the zero section. For example, $\mathbb{R}_{0}^{N}:=\mathbb{R}^{N} \backslash\{0\}, T_{0}^{*} M:=T^{*} M \backslash\{0\}$, etc.

\subsection{Fourier integral operators}

Here we recall some basic facts from the theory of Fourier integral operators (see 1113 ).

Let $X$ be a smooth closed manifold of dimension $n$. Denote by $\left(x, x^{\prime}\right)$ local coordinates on $X \times X$, and denote by $\left(x, p ; x^{\prime}, p^{\prime}\right)$ the corresponding coordinates on $T^{*}(X \times X)$. Fix the symplectic form $\omega_{X \times X}=d x \wedge d p-d x^{\prime} \wedge d p^{\prime}$ on $T^{*}(X \times X)$.

Definition 2.1. A function $\phi\left(x, x^{\prime}, \theta\right) \in C^{\infty}(\Gamma)$ defined on some open cone $\Gamma \subset$ $X \times X \times \mathbb{R}_{0}^{N}$ is called a phase function if

1) it is real-valued and homogeneous of degree 1 with respect to $\theta$-variables.

$2)$ its gradient $\partial_{x, x^{\prime}, \theta} \phi$ vanishes nowhere on $\Gamma$.

A phase function $\phi\left(x, x^{\prime}, \theta\right)$ is said to be clean, if, in addition,

3) the set

$$
C_{\phi}=\left\{\left(x, x^{\prime}, \theta\right) \in \Gamma \mid \partial_{\theta} \phi=0\right\}
$$

is a smooth conic manifold and the $N \times(2 n+N)$-matrix

$$
\nabla\left(\partial_{\theta} \phi\right)=\left(\begin{array}{lll}
\partial_{\theta x}^{2} \phi & \partial_{\theta x^{\prime}}^{2} \phi & \partial_{\theta \theta}^{2} \phi
\end{array}\right)
$$

is of constant $\operatorname{rank} \operatorname{rk}\left(\partial_{\theta x} \phi\right)=N-e$ on $C_{\phi}$ with

$$
e=\operatorname{dim} C_{\phi}-2 \operatorname{dim} X \text {. }
$$

The set $C_{\phi}$ is called the critical set of $\phi$, and the number $e$ is called the excess of $\phi$. A phase function is nondegenerate if its excess $e=0$.

For brevity, from now on we do not mention the domain cone $\Gamma$ explicitly and consider $\phi$ as a function on the entire space $X \times X \times \mathbb{R}_{0}^{N}$ (still tacitly assuming it is defined on some open cone). 
Definition 2.2. A smooth submanifold $\Lambda \subset T_{0}^{*}(X \times X)$ is said to be associated with a phase function $\phi$ (we also say that $\Lambda$ is parametrized by $\phi$ ) if in some conic neighbourhood it is defined as the range of the critical set $C_{\phi}$ under the map

$$
\gamma_{\phi}: X \times X \times \mathbb{R}_{0}^{N} \longrightarrow T_{0}^{*}(X \times X), \quad\left(x, x^{\prime}, \theta\right) \longmapsto\left(x, \partial_{x} \phi ; x^{\prime},-\partial_{x^{\prime}} \phi\right),
$$

that is, $\gamma_{\phi}\left(C_{\phi}\right)$ is an open subset of $\Lambda$.

It turns out (see, for example, [11]) that given a clean phase function $\phi$, the set $\gamma_{\phi}\left(C_{\phi}\right)$ is an immersed conic submanifold in $T_{0}^{*}(X \times X)$, and, moreover, it is Lagrangian with respect to the form $\omega_{X \times X}$. Furthermore, in this case $\gamma_{\phi}: C_{\phi} \rightarrow \Lambda$ is a fibration with fibers of dimension $e$ (and it is a local diffeomorphism when $e=0$ ). We call this map the parametrization of $\Lambda$ by $\phi$.

Definition 2.3. A Fourier integral operator $\Phi=\Phi(\Lambda)$, associated with a conic Lagrangian submanifold $\Lambda \subset T_{0}^{*}(X \times X)$, is a linear operator $C_{0}^{\infty}(X) \rightarrow \mathcal{D}^{\prime}(X)$ whose Schwartz kernel $K_{\Phi} \in \mathcal{D}^{\prime}(X \times X)$ is locally of the form

$$
K_{\Phi}\left(x, x^{\prime}\right)=(2 \pi)^{-(n+N) / 2+e / 2} \int e^{i \phi\left(x, x^{\prime}, \theta\right)} a\left(x, x^{\prime}, \theta\right) d \theta .
$$

Here $\phi\left(x, x^{\prime}, \theta\right) \in C^{\infty}\left(X \times X \times \mathbb{R}_{0}^{N}\right)$ is a clean phase function of excess $e$ parametrizing $\Lambda, a\left(x, x^{\prime}, \theta\right) \in S^{d+(n-N-e) / 2}\left(X \times X \times \mathbb{R}^{N}\right)$ is an amplitude (a function from Hörmander's symbol class), the number $d$ is called the order of $\Phi$ (we write ord $\Phi=$ $d$ ). It is assumed that the support of $a$ is contained in the domain of $\phi$. The integral is defined in the sense of distributions (as an oscillatory integral).

Recall that the kernel (2.3) does not depend on the choice of $\phi$ modulo smooth functions (if one takes an appropriate amplitude), provided that $\phi$ parametrizes $\Lambda$ and the support of $a$ is sufficiently small. Thus $\Phi(\Lambda)$ does not depend on the way its Schwartz kernel is written down modulo smoothing operators, but on the underlying Lagrangian submanifold $\Lambda$ (in particular we can always assume that $\phi$ is nondegenerate, since any Lagrangian manifold admits a parametrization by such a phase function). See the details in [12,13].

Now recall that distributions of the form (2.3) admit a special representation in the framework of the theory of Maslov canonical operator [14, 15]). Namely, let the following collection of coordinate functions

$$
w=\left(x_{I}, p_{\bar{I}} ; x_{I^{\prime}}^{\prime}, p_{\bar{I}^{\prime}}^{\prime}\right),
$$

where $I, I^{\prime} \subset\{1, \ldots, n\}, \bar{I}=\{1, \ldots, n\} \backslash I, \bar{I}^{\prime}=\{1, \ldots, n\} \backslash I^{\prime}$, define a local coordinate system in some conic neighbourhood in $\Lambda$ (canonical coordinates on $\Lambda$ ). Then there is a smooth homogeneous function $S(w)$ of degree 1 on this neighbourhood (a generating function of $\Lambda$ ) such that $\Lambda$ in the coordinates $\left(x, p ; x^{\prime}, p^{\prime}\right)$ is defined by the equations

$$
x_{\bar{I}}=-\frac{\partial S(w)}{\partial p_{\bar{I}}}, \quad p_{I}=\frac{\partial S(w)}{\partial x_{I}}, \quad x_{\bar{I}^{\prime}}^{\prime}=\frac{\partial S(w)}{\partial p_{\bar{I}^{\prime}}^{\prime}}, \quad p_{I^{\prime}}^{\prime}=-\frac{\partial S(w)}{\partial x_{I^{\prime}}^{\prime}},
$$

where $w \in \Lambda$ is defined by (2.4). In this case the kernel (2.3) modulo smooth functions can be expressed as

$$
\begin{aligned}
K_{\Phi}\left(x, x^{\prime}\right)=\mathcal{F}_{p_{\bar{I}} \rightarrow x_{\bar{I}}}^{-1} \mathcal{F}_{p_{\bar{I}^{\prime}}^{\prime} \rightarrow x_{\bar{I}^{\prime}}^{\prime}} b(w) & = \\
& =(2 \pi)^{-\left(|\bar{I}|+\left|\bar{I}^{\prime}\right|\right) / 2} \iint e^{i S(w)+i p_{\bar{I}} x_{\bar{I}}-i p_{\bar{I}^{\prime}}^{\prime} x_{\bar{I}^{\prime}}^{\prime}} b(w) d p_{\bar{I}} d p_{\bar{I}^{\prime}}^{\prime}
\end{aligned}
$$


where $b \in S^{d+\left(n-|\bar{I}|-\left|\bar{I}^{\prime}\right|\right) / 2}(\Lambda)$, and $\mathcal{F}, \mathcal{F}^{-1}$ stand for the direct and inverse Fourier transforms, respectively. See also Proposition 3.7 below.

Lastly, we recall the notion of clean intersection of manifolds (see [11]).

Definition 2.4. Let $M_{3}$ be a manifold and let $M_{1}$ and $M_{2}$ be its submanifolds. The intersection $M_{1} \cap M_{2} \subset M_{3}$ is said to be clean if it is a submanifold in $M_{3}$ and for any point $\nu \in M_{1} \cap M_{2}$ we have

$$
T_{\nu}\left(M_{1} \cap M_{2}\right)=T_{\nu} M_{1} \cap T_{\nu} M_{2}
$$

\subsection{Traces of operators and traces of Lagrangian manifolds}

Let $i: X \hookrightarrow M$ be a smooth embedding of closed manifolds. Let $(x, y)$ be local coordinates on $M$ and let $X$ be defined in these coordinates by the equations $X=$ $\{y=0\}$. Denote by $(x, y, p, q)$ the corresponding coordinates on $T^{*} M$ and by

$$
\left(x, y, p, q ; x^{\prime}, y^{\prime}, p^{\prime}, q^{\prime}\right)
$$

the corresponding coordinates on $T^{*}(M \times M)$. Fix the symplectic form $\omega_{M \times M}$ on $T^{*}(M \times M)$ of the form

$$
\omega_{M \times M}=d x \wedge d p+d y \wedge d q-d x^{\prime} \wedge d p^{\prime}-d y^{\prime} \wedge d q^{\prime} .
$$

The embedding $i$ induces two special operators, namely the boundary operator $i^{*}$ and the coboundary operator $i_{*}$ (see [6]). The first one is an operator of restriction to the submanifold and the second one acts in a dual manner. More explicitly, in the above local coordinates these operators are defined as follows

$$
\begin{array}{ll}
i^{*}: H^{s}(M) \longrightarrow H^{s-\nu / 2}(X), & u(x, y) \longmapsto u(x, 0), \\
i_{*}: H^{-s+\nu / 2}(X) \longrightarrow H^{-s}(M), & u(x) \longmapsto u(x) \otimes \delta_{X}(y),
\end{array}
$$

where $\nu=\operatorname{codim}_{M} X$, and $\delta_{X}(y)$ stands for the Dirac delta-function localized at $X$. Both operators are continuous in the specified Sobolev spaces, provided that $s-\nu / 2>0$.

Let $\Phi$ be an operator on the ambient manifold $M$.

Definition 2.5. The trace $i^{!}(\Phi)$ of $\Phi$ on $X$ is the composition (see [1, 2])

$$
i^{!}(\Phi)=i^{*} \Phi i_{*}
$$

Remark 2.6. Clearly, the trace $i^{!}(\Phi)$ is an operator on the submanifold $X$. Note that the requirement $s-\nu / 2>0$, which limits the orders of the Sobolev spaces in (2.8), suggests that the composition (2.5) is not always well-defined. Namely, $\Phi$ should be a continuous operator in the spaces $H^{s}(M) \rightarrow H^{s-d}(M)$, where

$$
s<-\nu / 2, \quad s-d-\nu / 2>0 .
$$

In this case the trace $i^{!}(\Phi)$ is a continuous operator in the spaces

$$
i^{!}(\Phi): H^{s+\nu / 2}(X) \longrightarrow H^{s-d-\nu / 2}(X) .
$$

Now let $\Lambda$ be a submanifold in $T_{0}^{*}(M \times M)$. 
Definition 2.7. The trace $i^{!}(\Lambda)$ of $\Lambda$, associated to the embedding $i: X \hookrightarrow M$, is the set

$$
i^{!}(\Lambda)=\pi_{X \times X}\left(\left.\Lambda\right|_{X \times X}\right),
$$

where $\left.\Lambda\right|_{X \times X}$ is the intersection

$$
\left.\Lambda\right|_{X \times X}=\left.\Lambda \cap T^{*}(M \times M)\right|_{X \times X}
$$

and

$$
\pi_{X \times X}:\left.T^{*}(M \times M)\right|_{X \times X} \longrightarrow T^{*}(X \times X)
$$

stands for the projection induced by the embedding $i \times i: X \times X \hookrightarrow M \times M$.

Note that $i^{!}(\Lambda)$ is a subset in $T^{*}(X \times X)$.

\section{Traces of Fourier integral operators}

\subsection{The main theorem}

Here we state the main result of the present paper.

Theorem 3.1. Let $\Phi=\Phi(\Lambda): H^{s}(M) \rightarrow H^{s-d}(M)$ be a FIO associated with a Lagrangian submanifold $\Lambda \subset T_{0}^{*}(M \times M)$, where $s$ and d satisfy the inequalities (2.9). Let the following conditions hold:

1) the intersection (2.7) is clean;

2) one has $\Lambda \cap N^{*}(X \times X)=\emptyset$, where $N^{*}(X \times X)$ is the conormal bundle of $X \times X \subset M \times M$.

Then $i^{!}(\Lambda)$ is an immersed conic Lagrangian submanifold in $T_{0}^{*}(X \times X)$, and $i^{!}(\Phi)$ is a FIO associated with it:

$$
i^{!}(\Phi(\Lambda))=\Phi\left(i^{!}(\Lambda)\right)
$$

The order of $i^{!}(\Phi)$ is given by

$$
\operatorname{ord} i !(\Phi)=\operatorname{ord} \Phi-\operatorname{dim} X+\frac{1}{2} \operatorname{codim} X+\left.\frac{1}{2} \operatorname{dim} \Lambda\right|_{X \times X} .
$$

Proof. First of all, the limitations on the action of $\Phi$ in Sobolev spaces guarantee that $i^{!}(\Phi)$ is well-defined (see Remark 2.6). Next, it clearly suffices to prove (3.1) in local coordinates, so we can restrict our attention to some small conic neighbourhood $U \subset$ $T^{*}(M \times M)$ (with nonempty intersection with $\left.\left.T^{*}(M \times M)\right|_{X \times X}\right)$ and assume that $\Lambda$ is associated with some nondegenerate phase function in this neighbourhood. For brevity, from now on we identify all the manifolds under consideration with their neighbourhoods corresponding to $U$ : for example, we write $T^{*}(M \times M)$ instead of $U$, $\Lambda$ instead of $\Lambda \cap U$, etc. Let us also assume that $U$ is equipped with local coordinates of the form (2.7).

Under the above assumptions, we may consider $\Phi$ as an integral operator with Schwartz kernel

$$
K_{\Phi}\left(x, y, x^{\prime}, y^{\prime}\right)=(2 \pi)^{-(\operatorname{dim} M+N) / 2} \int e^{i \phi\left(x, y, x^{\prime}, y^{\prime}, \theta\right)} a\left(x, y, x^{\prime}, y^{\prime}, \theta\right) d \theta
$$


where $\phi \in C^{\infty}\left(M \times M \times \mathbb{R}_{0}^{N}\right)$ is a nondegenerate phase function, which parameterizes $\Lambda, a \in S^{d+(\operatorname{dim} M-N) / 2}\left(M \times M \times \mathbb{R}^{N}\right)$ is an amplitude, and $d=\operatorname{ord} \Phi$. Next, by a straightforward computation, we see that the trace $i^{!}(\Phi(\Lambda))$ is an integral operator with Schwartz kernel

$$
K_{i^{!}(\Phi)}\left(x, x^{\prime}\right)=\left.(2 \pi)^{-(\operatorname{dim} M+N) / 2} \int e^{\left.i \phi\right|_{X \times X}\left(x, x^{\prime}, \theta\right)} a\right|_{X \times X}\left(x, x^{\prime}, \theta\right) d \theta,
$$

where

$$
\left.\phi\right|_{X \times X}\left(x, x^{\prime}, \theta\right)=\phi\left(x, 0, x^{\prime}, 0, \theta\right),\left.\quad a\right|_{X \times X}\left(x, x^{\prime}, \theta\right)=a\left(x, 0, x^{\prime}, 0, \theta\right) .
$$

Note that $\left.a\right|_{X \times X}$ is an amplitude of the same order as $a$, i.e.

$$
a\left(x, 0, x^{\prime}, 0, \theta\right) \in S^{d+(\operatorname{dim} M-N) / 2}\left(X \times X \times \mathbb{R}^{N}\right) .
$$

Thus it is enough to prove that $\left.\phi\right|_{X \times X}$ is a clean phase function associated with $i^{!}(\Lambda)$. This will imply (3.1).

Step 1. Parametrization of $i^{!}(L)$. Let us show that $\left.\phi\right|_{X \times X}$ parameterizes $i^{!}(\Lambda)$ in the sense of Definition 2.2. We start from recalling the properties of $\phi$.

Since $\phi$ is nondegenerate, its critical set

$$
C_{\phi}=\left\{\left(x, y, x^{\prime}, y^{\prime}, \theta\right) \mid \partial_{\theta} \phi=0\right\} \subset M \times M \times \mathbb{R}_{0}^{N}
$$

is a smooth manifold of dimension $\operatorname{dim} C_{\phi}=2 \operatorname{dim} M$, and $\Lambda$ is the range of this submanifold under the map

$$
\begin{aligned}
\gamma_{\phi}: M \times M \times \mathbb{R}_{0}^{N} & \longrightarrow T_{0}^{*}(M \times M), \\
\left(x, y, x^{\prime}, y^{\prime}, \theta\right) & \longmapsto\left(x, y, \partial_{x} \phi, \partial_{y} \phi ; x^{\prime}, y^{\prime},-\partial_{x^{\prime}} \phi,-\partial_{y^{\prime}} \phi\right) .
\end{aligned}
$$

Furthermore, we can assume that the restriction of $\gamma_{\phi}$ to $C_{\phi}$ defines a diffeomorphism

$$
\gamma_{\phi}: C_{\phi} \stackrel{\simeq}{\longrightarrow} \Lambda
$$

This situation transfers to $\left.\phi\right|_{X \times X}$ as follows. First, the critical set of $\left.\phi\right|_{X \times X}$ is of the form

$$
C_{\left.\phi\right|_{X \times X}}=\left\{\left(x, x^{\prime}, \theta\right) \mid \partial_{\theta}\left(\left.\phi\right|_{X \times X}\right)=0\right\} \subset X \times X \times \mathbb{R}_{0}^{N} .
$$

Note that

$$
C_{\left.\phi\right|_{X \times X}}=C_{\phi} \cap X \times X \times \mathbb{R}_{0}^{N}
$$

and

$$
\left.\Lambda\right|_{X \times X}=\gamma_{\phi}\left(C_{\left.\phi\right|_{X \times X}}\right) .
$$

Now, since the intersection (2.7) is clean, $\left.\Lambda\right|_{X \times X}$ is a submanifold in $\Lambda$; hence, reverting the diffeomorphism (3.6), we deduce that $C_{\left.\phi\right|_{X \times X}}$ is a submanifold in $C_{\phi}$. (More precisely, $\gamma_{\phi}$ restricted to $C_{\left.\phi\right|_{X \times X}}$ defines a diffeomorphism $\left.C_{\left.\phi\right|_{X \times X}} \rightarrow \Lambda\right|_{X \times X}$.)

Second, the parametrization map corresponding to $\left.\phi\right|_{X \times X}$ is

$$
\begin{aligned}
\gamma_{\left.\phi\right|_{X \times X}}: X \times X \times \mathbb{R}_{0}^{N} & \longrightarrow T_{0}^{*}(X \times X), \\
\left(x, x^{\prime}, \theta\right) & \longmapsto\left(x, \partial_{x}\left(\left.\phi\right|_{X \times X}\right) ; x^{\prime},-\partial_{x^{\prime}}\left(\left.\phi\right|_{X \times X}\right)\right) .
\end{aligned}
$$

Evidently, it is a composition of the restriction $\gamma_{\phi}$ to $X \times X \times \mathbb{R}_{0}^{N}$ and the projection $\pi_{X \times X}$. Together with (3.7) and (3.8) this implies the identity

$$
i^{!}(\Lambda)=\gamma_{\left.\phi\right|_{X \times X}}\left(C_{\left.\phi\right|_{X \times X}}\right) .
$$


Thus $\left.\phi\right|_{X \times X}$ is associated with $i^{!}(\Lambda)$ in the sense of Definition 2.2, as desired.

Summarising, we get the following commutative diagram:

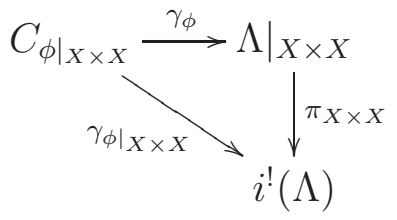

where the $\gamma_{\phi}$ is a diffeomorphism, and the $\pi_{X \times X}$ is a smooth map of constant rank.

Corollary 3.2. The set $i !(\Lambda)$ is an immersed conic submanifold in $T_{0}^{*}(X \times X)$.

Proof. From the commutativity of the diagram (3.10) we see that $\gamma_{\left.\phi\right|_{X \times X}}$ is a smooth map of constant rank (equal to the rank of $\pi_{X \times X}$ ), hence its image $i^{!}(\Lambda)$ is an immersed submanifold in $T^{*}(X \times X)$. The fact that it does not intersect the zero section of $T^{*}(X \times X)$ follows from the hypothesis 2$)$ of the current theorem. Indeed, let $\{0\} \subset T^{*}(X \times X)$ be the zero section. Then its preimage $\left[\pi_{X \times X}\right]^{-1}(\{0\})$ under the projection $\pi_{X \times X}$ is precisely the conormal bundle of $X \times X \hookrightarrow M \times M$,

$$
\left[\pi_{X \times X}\right]^{-1}(\{0\})=N^{*}(X \times X) .
$$

So $\Lambda \cap N^{*}(X \times X)=\emptyset$ implies $i^{!}(\Lambda) \cap\{0\}=\emptyset$. Corollary 3.2 follows.

On the next step we study the critical set of $\left.\phi\right|_{X \times X}$.

Step 2. Properties of $C_{\phi_{\mid X \times X}}$. Here we show that the intersection (2.7) transfers to the "parameter space" $M \times M \times \mathbb{R}_{0}^{N}$ and remains clean. We start from the subspace $\left.T_{0}^{*}(M \times M)\right|_{X \times X}$.

Lemma 3.3. One has

$$
\left.\gamma_{\phi}\left(X \times X \times \mathbb{R}_{0}^{N}\right) \subseteq T_{0}^{*}(M \times M)\right|_{X \times X} .
$$

Proof. This follows immediately from a direct computation.

Now we can describe $C_{\left.\phi\right|_{X \times X}}$.

Lemma 3.4. The set $C_{\left.\phi\right|_{X \times X}}$ is a submanifold in $M \times M \times \mathbb{R}_{0}^{N}$, and for any point $\nu \in C_{\left.\phi\right|_{X \times X}}$ we have

$$
T_{\nu} C_{\left.\phi\right|_{X \times X}}=T_{\nu} C_{\phi} \cap T_{\nu}\left(X \times X \times \mathbb{R}_{0}^{N}\right) .
$$

Remark 3.5. In other words, Lemma 3.4 claims that the intersection (3.7) is clean.

Proof. We have already seen that $C_{\left.\phi\right|_{X \times X}}$ is a submanifold in $C_{\phi}$. Since $C_{\phi}$ is a submanifold in $M \times M \times \mathbb{R}_{0}^{N}$, it follows that $C_{\left.\phi\right|_{X \times X}}$ is a submanifold in $M \times M \times \mathbb{R}_{0}^{N}$, as claimed. It remains to prove (3.11).

Let $\nu \in C_{\left.\phi\right|_{X \times X}}$ be fixed. Then (3.7) implies the inclusion

$$
T_{\nu} C_{\left.\phi\right|_{X \times X}} \subseteq T_{\nu} C_{\phi} \cap T_{\nu}\left(X \times X \times \mathbb{R}_{0}^{N}\right),
$$

hence, in order to establish (3.11), it suffices to obtain the inverse inclusion. Now consider the linear map

$$
d \gamma_{\phi}: T_{\nu}\left(M \times M \times \mathbb{R}_{0}^{N}\right) \longrightarrow T_{\gamma_{\phi}(\nu)}\left(T_{0}^{*}(M \times M)\right),
$$


induced by $\gamma_{\phi}$. Its restriction to $T_{\nu} C_{\phi} \subset T_{\nu}\left(M \times M \times \mathbb{R}_{0}^{N}\right)$ defines an isomorphism of vector spaces

$$
d \gamma_{\phi}: T_{\nu} C_{\phi} \stackrel{\simeq}{\longrightarrow} T_{\gamma_{\phi}(\nu)} \Lambda .
$$

Consequently, the desired inclusion is equivalent to the following:

$$
d \gamma_{\phi}\left(T_{\nu} C_{\phi} \cap T_{\nu}\left(X \times X \times \mathbb{R}_{0}^{N}\right)\right) \subseteq d \gamma_{\phi}\left(T_{\nu} C_{\left.\phi\right|_{X \times X}}\right) .
$$

On the other hand, by (3.8) we have

$$
d \gamma_{\phi}\left(T_{\nu} C_{\left.\phi\right|_{X \times X}}\right)=T_{\gamma_{\phi}(\nu)}\left(\left.\Lambda\right|_{X \times X}\right) .
$$

So it suffices to check that we have the inclusion

$$
d \gamma_{\phi}\left(T_{\nu} C_{\phi} \cap T_{\nu}\left(X \times X \times \mathbb{R}_{0}^{N}\right)\right) \subseteq T_{\gamma_{\phi}(\nu)}\left(\left.\Lambda\right|_{X \times X}\right) .
$$

Now note that (3.3) and (3.12) imply

$$
d \gamma_{\phi}\left(T_{\nu}\left(X \times X \times \mathbb{R}_{0}^{N}\right)\right) \subseteq T_{\gamma_{\phi}(\nu)}\left(\left.T_{0}^{*}(M \times M)\right|_{X \times X}\right), \quad d \gamma_{\phi}\left(T_{\nu} C_{\phi}\right)=T_{\gamma_{\phi}(\nu)} \Lambda .
$$

Therefore, we have

$$
\begin{aligned}
d \gamma_{\phi}\left(T_{\nu} C_{\phi} \cap T_{\nu}(X \times X \times\right. & \left.\left.\mathbb{R}_{0}^{N}\right)\right) \subseteq d \gamma_{\phi}\left(T_{\nu} C_{\phi}\right) \cap d \gamma_{\phi}\left(T_{\nu}\left(X \times X \times \mathbb{R}_{0}^{N}\right)\right) \subseteq \\
& \subseteq T_{\gamma_{\phi}(\nu)} \Lambda \cap T_{\gamma_{\phi}(\nu)}\left(\left.T_{0}^{*}(M \times M)\right|_{X \times X}\right)=T_{\gamma_{\phi}(\nu)}\left(\left.\Lambda\right|_{X \times X}\right),
\end{aligned}
$$

where the last equation holds because the intersection (2.7) is clean. It follows that (3.13) holds, and so Lemma 3.4 is proved.

Let us now study $\left.\phi\right|_{X \times X}$.

Step 3. Properties of $\left.\phi\right|_{X \times X}$.

Lemma 3.6. The function $\left.\phi\right|_{X \times X}$ is a clean phase function with excess

$$
e=\left.\operatorname{dim} \Lambda\right|_{X \times X}-2 \operatorname{dim} X .
$$

Proof. Let us check that $\left.\phi\right|_{X \times X}$ meets the requirements listed in Definition 2.1.

1) $\left.\phi\right|_{X \times X}$ is real-valued and homogeneous of degree 1 with respect to $\theta$-variables. This is obvious.

2) The gradient $\partial_{x, x^{\prime}, \theta}\left(\left.\phi\right|_{X \times X}\right)$ vanishes nowhere on $C_{\left.\phi\right|_{X \times X}}$. Indeed, otherwise the set (3.9) would have a nonempty intersection with the zero section $\{0\} \subset T^{*}(X \times X)$ contradicting Corollary 3.2 .

3) Lemma 3.4 implies that $\left.\phi\right|_{X \times X}$ has an excess in the sense of Definition 2.1. Indeed, a direct computation shows that (3.11) leads to the equality

$$
T_{\nu} C_{\left.\phi\right|_{X \times X}}=\operatorname{Ker} \Psi(\nu), \quad \forall \nu \in C_{\left.\phi\right|_{X \times X}},
$$

where $\Psi$ is a linear operator given by the $N \times(2 \operatorname{dim} X+N)$ matrix

$$
\left(\begin{array}{lll}
\partial_{\theta x}^{2}\left(\left.\phi\right|_{X \times X}\right) & \partial_{\theta x^{\prime}}^{2}\left(\left.\phi\right|_{X \times X}\right) & \partial_{\theta \theta}^{2}\left(\left.\phi\right|_{X \times X}\right)
\end{array}\right) .
$$

Consequently,

$$
\operatorname{dim} C_{\left.\phi\right|_{X \times X}}=2 \operatorname{dim} X+N-\operatorname{rk} \Psi .
$$

Therefore

$$
\operatorname{rk} \Psi=N-e, \quad e=\operatorname{dim} C_{\left.\phi\right|_{X \times X}}-2 \operatorname{dim} X .
$$

On the other hand, note that the matrix (3.15) is nothing but the matrix $\nabla\left(\left.\partial_{\theta} \phi\right|_{X \times X}\right)$ (see (2.1)), so (3.16) means that the number $e$ is the excess of $\left.\phi\right|_{X \times X}$ (compare (3.16) and (2.2)). Finally, by virtue of the diffeomorphism $\left.C_{\left.\phi\right|_{X \times X}} \simeq \Lambda\right|_{X \times X}$ (see (3.10)) we have $\operatorname{dim} C_{\phi}=\left.\operatorname{dim} \Lambda\right|_{X \times X}$, so (3.14) holds.

The properties 1)-2) mean that $\left.\phi\right|_{X \times X}$ is a phase function, and the property 3) means it is clean with excess (3.14). Lemma 3.6 is proved. 
Step 4. Conclusion. The above arguments show that the manifold $i^{!}(\Lambda)$ is associated with a clean phase function $\left.\phi\right|_{X \times X}$ of excess (3.14), and the map

$$
\gamma_{\left.\phi\right|_{X \times X}}: C_{\left.\phi\right|_{X \times X}} \longrightarrow i^{!}(\Lambda)
$$

defines the corresponding parametrization. It follows that $i !(\Lambda)$ is an immersed Lagrangian submanifold in $T_{0}^{*}(X \times X)$, and the expression (3.4) defines a kernel of a FIO associated with $i^{!}(\Lambda)$. The formula (3.2) follows directly from (3.5) and (3.14) (see Definition 2.3).

The proof of Theorem 3.1 is complete.

\subsection{Calculation of amplitude}

Now we refine Theorem 3.1 by representing the kernel (3.4) in the form of (2.6) for some amplitude $b(w)$ on $i^{!}(\Lambda)$.

At first we need to make some preparations. Assume that a local conic chart $U \subset T^{*}(M \times M)$ with coordinates (2.7) is chosen1, the conditions of Theorem 3.1 are fulfilled, and the kernel $K_{\Phi}$ of $\Phi$ is of the form (3.3). Then $i^{!}(\Lambda)$ is a Lagrangian submanifold, and we have two natural ways to describe it.

1. On the one hand, since $i^{!}(\Lambda)$ is Lagrangian, there is a collection of coordinate functions

$$
w=\left(x_{I}, p_{\bar{I}} ; x_{I^{\prime}}^{\prime}, p_{\bar{I}^{\prime}}^{\prime}\right),
$$

which defines a coordinate system on $i^{!}(\Lambda)$. Let such a collection be fixed, and let $S(w)$ be the corresponding generating function of $i^{!}(\Lambda)$. Then $i^{!}(\Lambda)$ is defined by the equations (2.5).

2. On the other hand, according to the proof of Theorem 3.1, $i^{!}(\Lambda)$ is associated with a clean phase function $\left.\phi\right|_{X \times X}$ of excess $e$ (the latter is defined by (3.14)). It follows that the parametrization (3.17) is a fibration whose fibers

$$
F_{w} \stackrel{\text { def }}{=}\left[\gamma_{\left.\phi\right|_{X \times X}}\right]^{-1}(w) \cap C_{\left.\phi\right|_{X \times X}}, \quad w \in i^{!}(\Lambda),
$$

are smooth $e$-dimensional manifolds. Moreover, it can be shown (see the procedure of elimination of excess described in [13]) that there is (possibly after a linear transformation of $\theta$-variables and provided that the neighbourhood $U$ is sufficiently small) a splitting

$$
\theta=\left(\theta^{\prime}, \theta^{\prime \prime}\right), \quad \theta^{\prime} \in \mathbb{R}^{N-e}, \theta^{\prime \prime} \in \mathbb{R}^{e},
$$

such that the variables $\theta^{\prime \prime}$ define local coordinates in the fibers of (3.17), and $\theta^{\prime} \neq 0$ for all $\left(x, x^{\prime}, \theta\right) \in C_{\left.\phi\right|_{X \times X}}$.

The next proposition connects two different expressions of the kernel $K_{i^{\prime}(\Phi)}$ corresponding to the two descriptions of $i^{!}(\Lambda)$ given above. Here for simplicity we assume that the amplitude $a$ in (3.3) is a classical symbol, i.e. it admits an asymptotic expansion in decreasing orders of homogeneity (see [16]). By $a_{0}$ we denote the leading term for $a$.

Proposition 3.7. Under the conditions of Theorem 3.1 the kernel $K_{i^{!}(\Phi)}$ modulo smooth functions is of the form (2.6), where $b$ is a classical symbol and its leading

\footnotetext{
${ }^{1}$ As before, we write $T^{*}(M \times M)$ instead of $U, \Lambda$ instead of $\Lambda \cap U$, etc.
} 
term is given by

$$
\begin{aligned}
b_{0}(w)=(2 \pi)^{-(\operatorname{dim} M+N-e) / 2} \int_{F_{w}} e^{\frac{i \pi}{4} \operatorname{sgn} H_{w, \theta^{\prime \prime}}\left(\widetilde{x}_{\bar{I}}, \widetilde{x}_{\bar{I}^{\prime}}^{\prime}, \widetilde{\theta}^{\prime}\right)} \times \\
\times\left.\left|\operatorname{det} H_{w, \theta^{\prime \prime}}\left(\widetilde{x}_{\bar{I}}, \widetilde{x}_{\bar{I}^{\prime}}^{\prime}, \widetilde{\theta}^{\prime}\right)\right|^{-1 / 2} a_{0}\right|_{X \times X}\left(x_{I}, \widetilde{x}_{\bar{I}}, x_{I^{\prime}}^{\prime}, \widetilde{x}_{\bar{I}^{\prime}}^{\prime}, \widetilde{\theta}^{\prime}, \theta^{\prime \prime}\right) d \theta^{\prime \prime},
\end{aligned}
$$

where $w \in i^{!}(\Lambda)$ is given by (13.18), $e=\left.\operatorname{dim} \Lambda\right|_{X \times X}-2 \operatorname{dim} X, H_{w, \theta^{\prime \prime}}$ is the Hessian matrix of $\left.\phi\right|_{X \times X}\left(x, x^{\prime}, \theta\right)$ with respect to the variables $\left(x_{\bar{I}}, x_{\bar{I}^{\prime}}, \theta^{\prime}\right)$, and the point $\left(\widetilde{x}_{\bar{I}}, \widetilde{x}_{\bar{I}^{\prime}}^{\prime}, \widetilde{\theta}^{\prime}\right)$ is determined by $w$ and $\theta^{\prime \prime}$ via the equation

$$
\gamma_{\left.\phi\right|_{X \times X}}\left(x_{I}, \widetilde{x}_{\bar{I}}, x_{I^{\prime}}^{\prime}, \widetilde{x}_{\bar{I}^{\prime}}^{\prime}, \widetilde{\theta}^{\prime}, \theta^{\prime \prime}\right)=w .
$$

Proof. We want to find an amplitude $b$ on $i^{!}(\Lambda)$ such that the expressions (3.4) and (2.6) define the same distribution modulo smooth functions. Applying the composition of Fourier transforms $\mathcal{F}_{x_{\bar{I}} \rightarrow p_{\bar{I}}} \mathcal{F}_{x_{\bar{I}^{\prime}}^{\prime} \rightarrow p_{\bar{I}^{\prime}}^{\prime}}^{-1}$ to both of these expressions, we get

$$
\begin{aligned}
b(w)=(2 \pi)^{-(\operatorname{dim} M+N) / 2-\left(|\bar{T}|+\left|\bar{I}^{\prime}\right|\right) / 2} \int e^{i\left[\left.\phi\right|_{X \times X}\left(x, x^{\prime}, \theta\right)-S(w)-p_{\bar{I}} x_{\bar{I}}+p_{\bar{I}^{\prime}}^{\prime} x_{\bar{I}^{\prime}}^{\prime}\right]} \times \\
\times\left. a\right|_{X \times X}\left(x, x^{\prime}, \theta\right) d x_{\bar{I}} d x_{\bar{I}^{\prime}}^{\prime} d \theta .
\end{aligned}
$$

Now the rest of the proof is a computation of the integral (3.19) via the method of stationary phase. We refer the reader to the proof of Proposition 25.1.5' in [12], where an analogous integral was considered, and only sketch out some basic points of this computation.

Step 1. Determining the stationary points. Since the integral (3.19) depends on the parameter $w \in i^{!}(\Lambda)$ given by the coordinate functions (3.18), let us assume that this parameter is fixed. A straightforward calculation shows that a value of the collection $\left(x_{\bar{I}}, x_{\bar{I}^{\prime}}^{\prime}, \theta\right)$ defines a stationary point for the integral (3.19) if the corresponding value of the collection $\left(x, x^{\prime}, \theta\right)$ defines a point in $F_{w}$. Thus we may assume that the integration is being performed over some neighbourhood of the set

$$
\widetilde{F}_{w} \stackrel{\text { def }}{=}\left\{\left(x_{\bar{I}}, x_{\bar{I}^{\prime}}^{\prime}, \theta\right) \mid\left(x, x^{\prime}, \theta\right) \in F_{w}\right\} .
$$

Step 2. Reducing to a repeated integral. Using the splitting $\theta=\left(\theta^{\prime}, \theta^{\prime \prime}\right)$ and the fact that $\theta^{\prime \prime}$ define local coordinates in $F_{w}$, we can rewrite the integral (3.19) in the form

$$
b(w)=\int_{F_{w}} c\left(w, \theta^{\prime \prime}\right) d \theta^{\prime \prime},
$$

where $c\left(w, \theta^{\prime \prime}\right)$ is given by

$$
\begin{aligned}
c\left(w, \theta^{\prime \prime}\right)=(2 \pi)^{-(\operatorname{dim} M+N) / 2-\left(|\bar{I}|+\left|\bar{I}^{\prime}\right|\right) / 2} \int_{V_{\theta^{\prime \prime}}} e^{i\left[\left.\phi\right|_{X \times X}\left(x, x^{\prime}, \theta^{\prime}, \theta^{\prime \prime}\right)-S(w)-p_{\bar{I}} x_{\bar{I}}+p_{\bar{I}^{\prime}}^{\prime} x_{\bar{I}^{\prime}}^{\prime}\right]} \times \\
\times\left. a\right|_{X \times X}\left(x, x^{\prime}, \theta^{\prime}, \theta^{\prime \prime}\right) d x_{\bar{I}} d x_{\bar{I}^{\prime}}^{\prime} d \theta^{\prime},
\end{aligned}
$$

where $V_{\theta^{\prime \prime}}$ is the set of all $\left(x_{\bar{I}}, x_{\bar{I}^{\prime}}^{\prime}, \theta^{\prime}\right)$ such that $\left(x_{\bar{I}}, x_{\bar{I}^{\prime}}^{\prime}, \theta\right)$ lie in a neighbourhood of $\widetilde{F}_{w}$. We claim that the integral (3.20) is actually over a bounded domain, therefore it converges. Indeed, since $\theta^{\prime} \neq 0$ for all $\left(x, x^{\prime}, \theta\right) \in F_{w} \subset C_{\left.\phi\right|_{X \times X}}$, the same remains 
true for all points in some conic neighbourhood $W$ of $F_{w}$. But then the values of $\left|\theta^{\prime}\right|$ for $\left(x, x^{\prime}, \theta\right) \in W$ can not be arbitrary small; since $W$ is conic it follows that $\left|\theta^{\prime \prime}\right|$ can not be arbitrary large. This means that the values of $\left|\theta^{\prime \prime}\right|$ are bounded for all points in $F_{w}$, as claimed.

Step 3. Calculating $c\left(w, \theta^{\prime \prime}\right)$. Now let us consider the integral (3.21) as depending on the parameter $\theta^{\prime \prime}$ and assume that the value $\theta^{\prime \prime}=$ const is fixed. The idea is to apply to (3.21) the method of stationary phase. We make the following observations.

1) The point $\left(x_{\bar{I}}, x_{\bar{I}^{\prime}}^{\prime}, \theta^{\prime}\right)$ is stationary for the integral (3.21) if $\left(x, x^{\prime}, \theta\right) \in F_{w} \cap$ $\left\{\theta^{\prime \prime}=\right.$ const $\}$. It follows that this point is unique, provided that the neighbourhood $U$ is sufficiently small.

2) The phase function of the integral (3.21) is given by

$$
\left.\left(x_{\bar{I}}, x_{\bar{I}^{\prime}}^{\prime}, \theta^{\prime \prime}\right) \longmapsto \phi\right|_{X \times X}\left(x, x^{\prime}, \theta^{\prime}, \theta^{\prime \prime}\right)-S(w)-p_{\bar{I}} x_{\bar{I}}+p_{\bar{I}^{\prime}}^{\prime} x_{\bar{I}^{\prime}}^{\prime} .
$$

Its Hessian matrix is of the form

$$
H_{w, \theta^{\prime \prime}}\left(x_{\bar{I}}, x_{\bar{I}^{\prime}}^{\prime}, \theta^{\prime}\right)=\left(\begin{array}{ccc}
\partial_{x_{\bar{I}} x_{\bar{I}}}^{2}\left(\left.\phi\right|_{X \times X}\right) & \partial_{x_{\bar{I}} x_{\bar{I}^{\prime}}^{\prime}}^{2}\left(\left.\phi\right|_{X \times X}\right) & \partial_{x_{\bar{I}} \theta^{\prime}}^{2}\left(\left.\phi\right|_{X \times X}\right) \\
\partial_{x_{\bar{I}^{\prime}}^{\prime} x_{\bar{I}}}^{2}\left(\left.\phi\right|_{X \times X}\right) & \partial_{x_{\bar{I}^{\prime}}^{\prime} x_{\bar{I}^{\prime}}^{\prime}}^{2}\left(\left.\phi\right|_{X \times X}\right) & \partial_{x_{\bar{I}^{\prime}}^{\prime} \theta^{\prime}}^{2}\left(\left.\phi\right|_{X \times X}\right) \\
\partial_{\theta^{\prime} x_{\bar{I}}}^{2}\left(\left.\phi\right|_{X \times X}\right) & \partial_{\theta^{\prime} x_{\bar{I}^{\prime}}^{\prime}}^{2}\left(\left.\phi\right|_{X \times X}\right) & \partial_{\theta^{\prime} \theta^{\prime}}^{2}\left(\left.\phi\right|_{X \times X}\right)
\end{array}\right)
$$

The next lemma shows that this matrix is nondegenerate at the stationary point.

Lemma 3.8. The matrix $H_{w, \theta^{\prime \prime}}\left(x_{\bar{I}}, x_{\bar{I}^{\prime}}^{\prime}, \theta^{\prime}\right)$ is nondegenerate for all $\left(x_{\bar{I}}, x_{\bar{I}^{\prime}}^{\prime}, \theta^{\prime}\right)$ such that $\left(x, x^{\prime}, \theta\right) \in F_{w} \cap\left\{\theta^{\prime \prime}=\right.$ const $\}$.

Proof. Consider the composition

$$
C_{\left.\phi\right|_{X \times X}} \cap\left\{\theta^{\prime \prime}=\mathrm{const}\right\} \longrightarrow i^{!}(\Lambda) \longrightarrow \mathbb{R}_{x_{I}}^{|I|} \times \mathbb{R}_{p_{\bar{I}}}^{|\bar{I}|} \times \mathbb{R}_{x_{I^{\prime}}^{\prime}}^{\left|I^{\prime}\right|} \times \mathbb{R}_{p_{\bar{I}^{\prime}}^{\prime}}^{\left|\bar{I}^{\prime}\right|}
$$

given by

$$
\begin{aligned}
\left(x_{I}, x_{\bar{I}}, x_{I^{\prime}}^{\prime}, x_{\bar{I}^{\prime}}^{\prime}, \theta\right) & \longmapsto\left(x, \partial_{x}\left(\left.\phi\right|_{X \times X}\right) ; x^{\prime},-\partial_{x^{\prime}}\left(\left.\phi\right|_{X \times X}\right)\right) \\
& \left(x_{I}, \partial_{x_{\bar{I}}}\left(\left.\phi\right|_{X \times X}\right) ; x_{I^{\prime}}^{\prime},-\partial_{x_{\bar{I}^{\prime}}^{\prime}}\left(\left.\phi\right|_{X \times X}\right)\right)
\end{aligned}
$$

(the first arrow is the parametrization $\gamma_{\left.\phi\right|_{X \times X}}$, and the second arrow is the coordinate map). By construction this composition is a diffeomorphism onto its image. We complete it to the map

$$
\left(x_{I}, x_{\bar{I}}, x_{I^{\prime}}^{\prime}, x_{\bar{I}^{\prime}}^{\prime}, \theta\right) \longmapsto\left(x_{I}, \partial_{x_{\bar{I}}}\left(\left.\phi\right|_{X \times X}\right) ; x_{I^{\prime}}^{\prime},-\partial_{{x_{\bar{I}}^{\prime}}^{\prime}}\left(\left.\phi\right|_{X \times X}\right) ; \partial_{\theta^{\prime}}\left(\left.\phi\right|_{X \times X}\right)\right) .
$$

Since $\partial_{\theta^{\prime}}\left(\left.\phi\right|_{X \times X}\right)=0$ on $C_{\left.\phi\right|_{X \times X}}$, it follows that this map has surjective differential for all $\left(x, x^{\prime}, \theta\right)$ such that $\left(x, x^{\prime}, \theta\right) \in C_{\left.\phi\right|_{X \times X}} \cap\left\{\theta^{\prime \prime}=\right.$ const $\}$. Consequently, the map

$$
\left(x_{\bar{I}}, x_{\bar{I}^{\prime}}^{\prime}, \theta^{\prime}\right) \longmapsto\left(\partial_{x_{\bar{I}}}\left(\left.\phi\right|_{X \times X}\right), \partial_{x_{\bar{I}^{\prime}}^{\prime}}\left(\left.\phi\right|_{X \times X}\right), \partial_{\theta^{\prime}}\left(\left.\phi\right|_{X \times X}\right)\right)
$$

has surjective differential for all $\left(x_{\bar{I}}, x_{\bar{I}^{\prime}}^{\prime}, \theta^{\prime}\right)$ such that $\left(x, x^{\prime}, \theta\right) \in F_{w} \cap\left\{\theta^{\prime \prime}=\right.$ const $\}$. Therefore its Jacobian matrix is nondegenerate at such points. But this Jacobian matrix is equal to $H_{w, \theta^{\prime \prime}}\left(x_{\bar{I}}, x_{\bar{I}^{\prime}}^{\prime}, \theta^{\prime}\right)$, and this proves the lemma.

3) Finally, it is easy to see that the value of the function (3.22) is zero at the stationary point.

Now, applying the method of stationary phase to $c\left(w, \theta^{\prime \prime}\right)$ and substituting it into (3.20), we obtain the desired formula for $b(w)$.

The proof of Proposition 3.7 is complete. 


\subsection{Application to quantized canonical transformations}

In this section we apply Theorem 3.1 to quantized canonical transformations.

Let us recall some basic definitions. Let $g: T_{0}^{*} M \rightarrow T_{0}^{*} M$ be a homogenous canonical transformation (i.e. a conic diffeomorphism preserving the symplectic form $\left.\omega_{M \times M}\right)$. Then its graph

$$
\text { graph } g=\left\{\left(g\left(w^{\prime}\right), w^{\prime}\right)\right\} \subset T_{0}^{*} M \times T_{0}^{*} M .
$$

is a Lagrangian submanifold in $T_{0}^{*}(M \times M)$. A FIO $\Phi=\Phi($ graph $g)$ associated with graph $g$ is called a quantized canonical transformation. One of the main features of these operators is that they are bounded in the whole scale of Sobolev spaces. Namely, $\Phi=\Phi($ graph $g)$ acts continuously in the spaces

$$
\Phi: H^{s}(M) \longrightarrow H^{s-\operatorname{ord} \Phi}(M) \quad \forall s .
$$

The next corollary is a particular case of Theorem 3.1.

Corollary 3.9. Let $\Phi=\Phi($ graph $g)$ be a quantized canonical transformation of order $\operatorname{ord} \Phi<-\operatorname{codim} X$. Let the canonical transformation $g$ satisfy the following conditions:

1) the intersection $\left.T_{0}^{*} M\right|_{X} \cap g\left(\left.T_{0}^{*} M\right|_{X}\right) \subset T_{0}^{*} M$ is clean;

2) one has $N_{0}^{*} X \cap g\left(N_{0}^{*} X\right)=\emptyset$, where $N^{*} X$ is the conormal bundle of $X \subset M$.

Then $i^{!}$(graph $\left.g\right)$ is an immersed Lagrangian submanifold in $T_{0}^{*}(X \times X)$, and $i^{!}(\Phi)$ is a FIO associated with it:

$$
i^{!}(\Phi(\operatorname{graph} g))=\Phi\left(i^{!}(\operatorname{graph} g)\right) .
$$

Proof. The requirement ord $\Phi<-\operatorname{codim} X$ guarantees that the trace $i^{!}(\Phi)$ is welldefined. Let us show that the conditions 1) and 2) in Corollary 3.9 imply the conditions 1) and 2) in Theorem 3.1.

Step 1. Condition 1). We are going to check that the intersection

$$
\left.(\operatorname{graph} g)\right|_{X \times X}=\left.\operatorname{graph} g \cap T_{0}^{*}(M \times M)\right|_{X \times X}
$$

is clean. To simplify the notation let us prove this fact in a slightly more abstract setting.

Lemma 3.10. Let $f: Y \rightarrow Y$ be a diffeomorphism between smooth manifolds, and let $Z \subset Y$ be a submanifold. If the intersection $Z \cap f(Z) \subset Y$ is clean then so is the intersection graph $f \cap Z \times Z \subset Y \times Y$.

Proof. 1) First, let us show that the set graph $f \cap Z \times Z$ is a submanifold in $Y \times Y$. Indeed, denote by $(f$, id $)$ the map

$$
(f, \mathrm{id}): Y \longrightarrow Y \times Y, \quad \nu \longmapsto(f(\nu), \nu) .
$$

This map is clearly a diffeomorphism $Y \rightarrow \operatorname{graph} f$, and, moreover,

$$
\text { graph } f \cap Z \times Z=(f, \text { id })\left[f^{-1}(Z \cap f(Z))\right] .
$$

Since the intersection $Z \cap f(Z)$ is clean, it is a submanifold in $Y$; hence, since $f$ is a diffeomorphism, $f^{-1}(Z \cap f(Z))$ is a submanifold in $Y$ as well. Using (3.23) we deduce from this that graph $f \cap Z \times Z$ is a submanifold in $Y \times Y$, as claimed. 
2) Let $\nu \in Z$ be a fixed point such that $f(v) \in Z$. We claim that the following holds:

$$
T_{f(\nu) \times \nu}(\operatorname{graph} f \cap Z \times Z)=T_{f(\nu) \times \nu}(\operatorname{graph} f) \cap T_{f(\nu) \times \nu}(Z \times Z) .
$$

Indeed, firstly note that

$$
T_{f(\nu) \times \nu}(\operatorname{graph} f)=\operatorname{graph} d f
$$

(by $d f$ we denote the linear map $T_{\nu} Y \rightarrow T_{f(\nu)} Y$ induced by $f$ ), so we have

$$
\begin{array}{r}
T_{f(\nu) \times \nu}(\operatorname{graph} f) \cap T_{f(\nu) \times \nu}(Z \times Z)=\operatorname{graph} d f \cap\left(T_{f(\nu)} Z \times T_{\nu} Z\right)= \\
=(d f, \mathrm{id})\left[(d f)^{-1}\left(T_{f(\nu)} Z \cap d f\left(T_{\nu} Z\right)\right)\right] .
\end{array}
$$

(The last equation is analogous to (3.23).) Secondly, since the intersection $Z \cap f(Z)$ is clean, we have

$$
T_{f(\nu)} Z \cap d f\left(T_{\nu} Z\right)=T_{f(\nu)} Z \cap T_{f(\nu)}(f(Z))=T_{f(\nu)}(Z \cap f(Z)) .
$$

Therefore

$$
\begin{array}{r}
T_{f(\nu) \times \nu}(\operatorname{graph} f) \cap T_{f(\nu) \times \nu}(Z \times Z)=(d f, \mathrm{id})\left[(d f)^{-1}\left(T_{f(\nu)} Z \cap d f\left(T_{\nu} Z\right)\right)\right]= \\
=(d f, \mathrm{id})\left[(d f)^{-1}\left(T_{f(\nu)}(Z \cap f(Z))\right]=d(f, \mathrm{id})\left[T_{\nu}\left(f^{-1}(Z \cap f(Z))\right)\right]=\right. \\
=T_{f(\nu) \times \nu}\left((f, \mathrm{id})\left[f^{-1}(Z \cap f(Z))\right]\right)=T_{f(\nu) \times \nu}(\operatorname{graph} f \cap Z \times Z) .
\end{array}
$$

(For the last equality we have used (3.23) directly.) Thus we have got (3.24).

Lemma 3.10 is proved.

Setting $Y=T_{0}^{*} M, Z=\left.T_{0}^{*} M\right|_{X}, f=g$ and applying Lemma 3.10, we see that the condition 1) of Theorem 3.1 is satisfied.

Step 2. Condition 2). Let us check that graph $g \cap N^{*}(X \times X)=\emptyset$. We use our abstract notation again.

Lemma 3.11. Let $f: Y \rightarrow Y$ is a diffeomorphism of smooth manifolds and let $Z \subset$ $Y$ be a submanifold. If $Z \cap f(Z)=\emptyset$ then graph $f \cap Z \times Z=\emptyset$.

Proof. Obviously follows from (3.23).

Setting $Y=T_{0}^{*} M, Z=N_{0}^{*} X, f=g$, and applying Lemma 3.11, we see that the condition 2) of Theorem 3.1 is satisfied as well.

Now Theorem 3.1 implies Corollary 3.9.

\section{References}

[1] S. Novikov and B. Sternin. Traces of elliptic operators on submanifolds and K-theory. Soviet Math. Dokl., 7(5):1373-1376, 1966.

[2] S. Novikov and B. Sternin. Elliptic operators and submanifolds. Soviet Math. Dokl., 7(6):1508-1512, 1966.

[3] A. Savin, E. Schrohe, and B. Sternin, Elliptic Operators Associated with Groups of Quantized Canonical Transformations. Bull. Math. Sci., 142, 2018. (In print). 
[4] B. Sternin, Relative elliptic theory and the Sobolev problem. DAN SSSR, 230(2):287-290, 1976. English transl.: Soviet Math. Dokl., 17, No. 5, 1976, 1306-1309.

[5] V. Nazaikinskii and B. Sternin, Relative elliptic theory. In Juan Gil, Thomas Krainer, and Ingo Witt, editors, Aspects of Boundary Problems in Analysis and Geometry, volume 151 of Operator Theory: Advances and Applications. Advances in Partial Differential Equations, pages 495-560, Basel-Boston-Berlin, 2004. Birkhäuser.

[6] B. Sternin. Elliptic and parabolic problems on manifolds with boundary consisting of components of different dimension. Trans. Moscow Math. Soc., 15:387429, 1966.

[7] B. Sternin and V. Shatalov, Relative elliptic theory and the Sobolev problems. Matematicheskii Sbornik, 187(11):115-144, 1996. English transl.: Sbornik: Mathematics 187, No. 11, 1996, p.1691-1720.

[8] A. Yu. Savin and B. Yu. Sternin. On nonlocal Sobolev problems. Dokl. Math., 88(1):421-424, 2013.

[9] A. Yu. Savin, and B. Yu. Sternin, Elliptic translators on manifolds with point singularities. Differ. Equations, 48(12):1577-1585, 2012.

[10] P. Sipailo, Traces of quantized canonical transformations localized at a finite set of points. Differ. Equations, 54(4), 2018. (In print).

[11] L. Hörmander, The Analysis of Linear Partial Differential Operators III. Springer-Verlag, Berlin Heidelberg New York Tokyo, 1985.

[12] L. Hörmander, The Analysis of Linear Partial Differential Operators IV. Springer-Verlag, Berlin Heidelberg New York Tokyo, 1985.

[13] F. Treves, Introduction to Pseudodifferential and Fourier Integral Operators Volume 2. Springer-Verlag, Berlin Heidelberg New York Tokyo, 1981.

[14] V. P. Maslov, Théorie des Perturbations et Méthod Asymptotiques. Dunod, Paris, 1972. French transl. from the Russian 1965 edition.

[15] V. Nazaikinskii, V. Oshmyan, B. Sternin, and V. Shatalov, Fourier integral operators and the canonical operator. Usp. Mat. Nauk, 36(2):81-140, 1981. English transl.: Russ. Math. Surv. 36, No. 2, 1981, 93-161.

[16] M. A. Shubin, Pseudodifferential Operators and Spectral Theory. SpringerVerlag, Berlin Heidelberg, 1985. 